\title{
LA PROMESA DE CONFIDENCIALIDAD: NUEVAS LUCES PARA LA INVESTIGACIÓN CIENTÍFICA Y LA PRÁCTICA PROFESIONAL EN SALUD MENTAL ${ }^{1}$
}

\author{
María Inés Winkler², Raúl Villarroel³, Diana Pasmanik² \\ Resumen: En este trabajo se revisita el concepto de confidencialidad, a la luz de nuevos desafíos que surgen de los avances \\ tecnológicos y de comunicaciones, y sus aplicaciones en la práctica profesional clínica y la investigación científica. Se fundamenta \\ el análisis en la presentación de antecedentes filosóficos, así como también la distinción y precisión respecto de conceptos \\ relacionados: intimidad, privacidad, anonimato, secreto profesional. Se hace un contrapunto entre privacidad, como derecho \\ de los pacientes y participantes de la investigación científica, y confidencialidad y secreto profesional, como deber profesional. \\ Se examinan nuevos retos a la confidencialidad en la práctica profesional y en la investigación científica en salud mental, como \\ por ejemplo la protección de la información en la ficha clínica electrónica. Se concluye enfatizando la incorporación del tema \\ en la reflexión ético-legal en la investigación y formación profesional.
}

Palabras clave: confidencialidad, privacidad, secreto profesional, antecedentes filosóficos, práctica clínica, investigación científica

\section{The promise of confidentiality: new lights for scientific research and professional practice in mental health}

\begin{abstract}
In this paper the concept of confidentiality is revisited in the light of the challenges arising from technological and communications advances and their applications in clinical practice and scientific research. The analysis is based on the presentation of philosophical background as well as the distinction and precision regarding related concepts: intimacy, privacy, anonymity, and professional secret. A counterpoint between privacy rights of patients and participants of scientific research and professional secrecy and confidentiality as professional duty is done. Also new challenges to privacy are developed in professional practice and scientific research in mental health, like the protection of confidentiality of the electronic clinical file. The authors conclude emphasizing the need to include the subject in the ethical and legal reflection in research and professional education.
\end{abstract}

Key words: confidentiality, privacy, professional secrecy, philosophical overview, professional practice, scientific research

A promessa de confidencialidade: novas luzes para a investigação científica e prática profissional em saúde mental

Resumo: Neste trabalho foi revisitado o conceito de confidencialidade, à luz dos novos desafios decorrentes de avanços tecnológicos na comunicação e suas aplicações na prática profissional clínica e na investigação científica. A análise foi fundamentada na apresentação dos antecedentes filosóficos, bem como também, na distinção e na precisão a respeito de conceitos relacionados: intimidade, privacidade, anonimato e sigilo profissional. Faz-se um contraponto entre a privacidade como um direito dos pacientes e de participantes de pesquisa científica e a confidencialidade e sigilo profissional, como um dever profissional. Desenvolve-se novos desafios à confidencialidade na prática profissional e na pesquisa científica em saúde mental, por exemplo, a proteção das informações em fichas clínicas electrônicas. Conclui-se enfatizando a importância da incorporação do tema na reflexão ética-legal na pesquisa e na formação profissional.

Palavras-chave: confidencialidade, privacidade, sigilo profissional, antecedentes filosóficos, prática clínica, pesquisa científica

\footnotetext{
${ }^{1}$ Trabajo financiado por PIA-CONICYT Proyecto SOC 1110, modalidad Anillos de Investigación en Ciencias sociales, y la Universidad de Santiago de Chile.

${ }^{2}$ Universidad de Santiago de Chile, Chile

Correspondencia: maria.winkler@usach.cl

${ }^{3}$ Universidad de Chile, Chile
} 
"Guardaré silencio sobre todo aquello que en mi profesión, o fuera de ella, oiga o vea en la vida de los hombres (sic) que no deba ser público, manteniendo estas cosas de manera que no se pueda hablar de ellas" (Juramento Hipocrático).

El presente artículo revisita el tema de la confidencialidad a la luz de algunos grandes cambios que la ciencia y las profesiones enfrentan, en un mundo que tiende a la globalización y que se "licúa" (1) como efecto de los avances en los medios de comunicación, redes sociales y tecnología en general. En efecto, los avances en informática han llevado a proponer el concepto de sociedad en riesgo(2), obligando a replantear el derecho a la intimidad(3).

Tales cambios provocan problemas desconocidos, que requieren nuevas lecturas de teorías, principios y normas éticas, constituidas en orientaciones apropiadas para la salvaguarda de los derechos de las personas que se relacionan con científicos y profesionales.

Durante los tres últimos años se ha ejecutado un proyecto de investigación, con el objetivo de construir conocimiento teórico y aplicado y formar investigadores en ética e interculturalidad. En el plano aplicado, el trabajo estuvo enfocado a la creación de instrumentos para la investigación y la generación y validación de estrategias y procedimientos educativos (Proyecto Anillos). A partir de los resultados del proyecto se hizo evidente la conveniencia de reflexionar acerca de las implicancias que tiene para el contexto actual de la investigación y la práctica profesional la dependencia creciente de nuevas tecnologías de las comunicaciones, el uso de equipos computacionales, internet y nuevas posibilidades de bases de datos y formas de registro digital. Ello conlleva uno de los máximos desafíos en la investigación y el ejercicio profesional hoy: la protección de la confidencialidad.

El objetivo entonces corresponde a una relectura del concepto de "confidencialidad", su distinción con conceptos afines y su aplicación a los nuevos contextos profesionales y científicos, particularmente en salud mental. Es indispensable para tal revisión referirse específicamente al proceso de consentimiento informado, tarea que exige, a su vez, un anclaje en teorías filosóficas que han contribuido a la comprensión de las diversas dimensiones y aristas involucradas en el tema, abordado en el siguiente apartado.

\section{Confidencialidad: antecedentes filosóficos para el consentimiento informado}

Se puede considerar con entera propiedad al "consentimiento informado" como una piedra angular de la ética de la investigación científica, que corresponde al proceso mediante el cual se garantiza por escrito que, después de haber recibido y comprendido toda la información necesaria y pertinente, quienes participan en un protocolo de investigación en calidad de sujetos de intervención han manifestado voluntariamente su intención de participar. Mediante dicha expresión de voluntad otorgan su autorización para que se les trate en la calidad señalada y sean objeto de procedimientos, tratamientos u otras acciones que, favoreciendo la generación de nuevos conocimientos, pudieran eventualmente suponer molestias, riesgos e inconvenientes, o hasta lesionar su salud y dignidad. Al mismo tiempo, el consentimiento informado debe dar cuenta de aquellos derechos que corresponden a las personas al actuar como sujetos de tales intervenciones, como de las obligaciones y las responsabilidades o deberes de acción que caben a quien investiga mediante tales actos y procesos.

Ahora bien, el consentimiento informado no debería concebirse únicamente como el recurso investigativo que establece la aceptación voluntaria de las personas participantes, bajo la sola expectativa de eliminar la posibilidad de que, después de realizado un acto, exista responsabilidad para el investigador que manipula la información relacionada. Además de ser esta una visión reduccionista, impide entender que para la propia ciencia puede haber una ventaja agregada si se parte de la confianza o la buena fe de los sujetos que son intervenidos buscando extraer de ellos la información pertinente a los fines de la investigación, más allá de la prevención de acciones futuras en contra del investigador(4).

Como señala Philip Kitcher, un elemento fundamental del consentimiento informado lo constituirá, por consiguiente, garantizar la confidencialidad y reserva que de la información obtenida a 
partir de los sujetos intervenidos haga quien la reciba, mediante ese acto de confianza que las personas llevan a cabo cuando aceptan participar en una investigación. Como es evidente, los términos "confianza" y "confidencialidad" están estrechamente vinculados por su familiaridad etimológica (del latín fides) y por la relación de reciprocidad que los articula en el horizonte de una "ciencia bien ordenada" $(5,6)$ por parámetros de referencia ética. El investigador está compelido a formular una promesa de confidencialidad ante quien le permite acceder a información sensible, muchas veces concerniente a aspectos de intimidad profunda y delicada que, de ser revelada en contextos ajenos a los de la investigación y con otros propósitos que no sean los declarados por la ciencia, podrían convertir la vida de las personas participantes en una verdadera fatalidad.

El cumplimiento o incumplimiento de la confidencialidad prometida respecto de la información entregada corrobora o defrauda, entonces, la confianza otorgada, equilibrando o desbalanceando una relación que, antes que todo, es una relación entre personas, quienes en la mayor parte de los casos son enteramente desconocidas entre sí hasta el momento del encuentro en la situación investigativa. De ahí la carga de valor ético que recae sobre el cumplimiento efectivo de la promesa de confidencialidad que cabe al científico, de cara a su deber de corresponder a la confianza que en su tarea deposita la sociedad a la que sirve.

Todo ello conduce a visualizar la íntima vinculación que a la vez existe entre confianza y "responsabilidad"(7). En este caso se trataría de aquello que algunos han querido cifrar como la "responsabilidad social de la ciencia", o la responsabilidad social del científico, si se prefiere $(8,9)$. El sujeto de la investigación confía en el investigador y lo presume capaz de cumplir su promesa de confidencialidad porque es responsable. La combinación, entonces, de confianza, confidencialidad y responsabilidad parece ser conditio sine qua non de una ciencia bien ordenada.

En este sentido, cabe recordar que en la primera sección de la Fundamentación de la Metafísica de las Costumbres Kant examina la posibilidad moral que pudiera estar contenida en la formulación de promesas falsas. Para ello presenta el ejemplo de quien pudiera verse apremiado por la necesidad $y$, en virtud de ello, decidiera pedir dinero en préstamo, a sabiendas de que luego no podrá pagarlo; aunque sí teniendo en cuenta que nadie le prestará nada como no prometa formalmente devolver lo pedido en determinado tiempo. Es por todos conocido que Kant finalmente concluye que el principio del egoísmo o de la propia utilidad podría ser quizá muy compatible con el bienestar subjetivo futuro del individuo, mas tal determinación nunca podría tener el valor de una ley natural universal, ni tampoco ser coherente consigo misma, por lo que siempre la hará ser contradictoria, puesto que universalidad de una ley semejante (que remita a la posibilidad de que alguien prometa a sabiendas de que no cumplirá) haría imposible la promesa misma y el fin que con ella es perseguido. Así, nadie creería en la promesa recibida y se burlarían de ella tomándola por engaño(10).

Habida cuenta del análisis kantiano, se podría agregar también una consideración acerca del hecho de que toda promesa está indisolublemente anexada a una disposición de confianza por parte de quien se constituye en su depositario, quien la acoge para sí y levanta expectativas de cumplimiento futuro respecto de lo que se le ha prometido. En relación con ello, subjetivamente establece un margen de probabilidades que le permite prever un estado de cosas por venir en el que se juega la satisfacción de su interés individual y el conjunto de implicancias que a este interés se vincula, albergando necesariamente en su cálculo racional de resultados la expectativa respecto de un eventual incumplimiento de aquello prometido. Leído en términos de las teorías de la elección racional, confiar en otra persona equivaldría a adoptar una decisión estratégica en vistas de maximizar la utilidad del propio comportamiento(11). Así, la confianza se perfila como un mecanismo para hacer frente a la libertad de otros y reducir la incertidumbre que ella nos genera, sobre todo cuando no podemos dilucidar si la interacción con los demás nos resultará beneficiosa y enfrentamos la disyuntiva de optar, o bien por la sospecha y la desconfianza absoluta, o por la confianza ciega e irracional ante ellos(11).

La confianza está siempre vinculada a una situación de riesgo eventual, de incerteza respecto de la ocurrencia de acontecimientos futuros respecto de los cuales no se tiene un seguro o absoluto con- 
trol; lo que es lo mismo que decir que la confianza constituye un problema para nuestra reflexión cuando existe la posibilidad de que podamos llegar a perder algo, como consecuencia de haber apostado por la buena fe de quien nos ha prometido algo.

Habiéndose perfilado la confianza, en tanto motivo de inquietud filosófica y científico-social, en tan variados dominios como los que van desde las teorías de la decisión racional a las de la neurobiología, constituiría un elemento clave en el establecimiento mismo de las promesas y permitiría la elucidación del estatuto moral del acto humano de prometer. A su vez, favorecería entender por qué la figura ontológico-moral de la promesa ha sido tan largamente debatida a lo largo de la historia del pensamiento. En tal sentido, podríamos pensar que el surgimiento de la civilización humana estaría ineludiblemente determinado por la posibilidad de "criar a un animal al que le resulte lícito hacer promesas", como dijera Nietzsche en La genealogía de la moral(12:5).

Hume intentará mostrar cómo pueden llegar los propietarios prudentes y cuidadosos de sus propios intereses a otorgar su confianza a desconocidos que les ofrecen servicios y bienes futuros mediante promesas o en virtud de un contrato. Para el filósofo escocés, disponerse confiadamente resulta una cuestión posible por la exclusiva razón de que, constituyéndonos en partes ligadas por una vinculación contractual, podremos rechazar todas las transacciones futuras a aquellos contratantes que no nos hayan cumplido. Y es que al decir "yo prometo" no solo invito a otro a depositar su confianza en mí, sino que, a la vez, lo invito a arruinar mi reputación si falto a lo que le he prometido(13).

Ser "leal", entonces, o cumplir las propias promesas, es una obligación moral, pero que resulta indiscernible del interés de conservación de la reputación personal que la constituye(14). Kant, por cierto, no admite la idea de que las promesas deban entenderse a partir de su asociación con amenazas implícitas. La obligación de cumplir las promesas constituye para él un imperativo categórico. El imperativo moral más absoluto referido a las promesas consiste precisamente en no hacer falsas promesas.
Más cercano temporalmente, Luhmann ha sostenido que el que da su confianza es un tomador de riesgo, que estima que el depositario de su confianza es un buen riesgo. Damos pruebas de seguridad cuando afrontamos situaciones que pueden ser peligrosas, y a las que tal vez no tenemos más remedio que enfrentarnos, de una manera u otra. Pero, tener confianza en una persona es siempre equivalente a aceptar un riesgo. Escogemos aventurar nuestro propio bienestar ante aquellos que demandan nuestra confianza(15). Luhmann afirma que la virtud de la confianza consiste en comportarse confiando en que los otros se comportarán de manera correcta, teniendo en cuenta la posibilidad de valorar dicha conducta en los demás y en uno mismo(15).

Retomando nuestro desarrollo inicial, deberíamos admitir que la estrecha vinculación que reúne a confianza, confidencialidad y responsabilidad se materializa en el ideal de una ciencia bien ordenada (well-ordered science), como aquel que ha descrito Kitcher, en el que los fines de la investigación científica terminan por acoplarse a los intereses de los ciudadanos establecidos mediante procedimientos democráticos, quedando así depositado el destino del conocimiento en un diálogo simétrico entre expertos de la ciencia y ciudadanos ilustra$\operatorname{dos}(5,6)$.

\section{Distinciones conceptuales: de la privacidad al secreto}

En el abordaje de los actuales desafíos en la práctica profesional y la investigación científica es imprescindible la precisión conceptual. Aunque ciertos trabajos distinguen entre "confidencial" y "anónimo", que usados como sinónimos no lo son(16), en relación con el manejo de información sensible es frecuente la aplicación indistinta de conceptos, relacionados entre sí pero con significados diferentes.

En primer lugar, la intimidad puede ser entendida como el derecho inherente a la persona que se refiere a lo más profundo de su ser; definida como "zona espiritual íntima y reservada de una persona o de un grupo, especialmente de la familia" (17:461). Para Bajo Fernández "es un ámbito personal, reservado a la curiosidad pública, absolutamente necesario para el desarrollo humano y 
donde enraizaría la personalidad"(18:599). Así, la intimidad correspondería a una capa profunda de la persona. La privacidad, por otra parte, se asentaría a un nivel menos profundo. López Díaz la define como "el derecho de los individuos, grupos o instituciones a determinar por ellos mismos, cuándo, cómo y cuánta información acerca de sí es comunicada a otros"(19:205). Entonces, lo privado es más amplio que lo íntimo.

En un análisis más sutil respecto del concepto de "privacidad", se ha propuesto desagregarla en tres categorías(20). La primera refiere al control sobre quién tiene acceso a la información; la segunda al control sobre quién tiene el derecho de observar a una persona cuando no está en un espacio público, y la tercera al control específico sobre las decisiones concernientes a uno mismo: por ejemplo, la decisión de las mujeres de tener o no hijos.

Así, el concepto de "privacidad" implica la libertad de la persona de elegir por sí mismo cuándo, en qué circunstancias y, en especial, hasta qué punto desea compartir o no sus actitudes, creencias y opiniones, con otros.

Siendo entonces la privacidad un derecho de las personas, en contraposición, la confidencialidad corresponde a una obligación de los/as profesionales. Definida como una "actitud que se le pide al sujeto conocedor del dato o hecho de la intimidad o privacidad de la persona" (21:20), supone "la obligación del profesional de mantener en secreto cualquier información proporcionada por el paciente, no pudiendo revelársela a un tercero sin su permiso específico" (22:74). La confidencialidad es un concepto próximo a la intimidad, de manera que cuanto más íntimo sea el dato, será necesario un mayor grado de confidencialidad.

La Asociación Americana de Psicología (APA, por sus iniciales en inglés) otorga a la confidencialidad la categoría de principio:

"Principio de la ética profesional que requiere que los profesionales de la salud mental o cuidado médico limiten la develación de la identidad de los pacientes, su condición o tratamiento, o cualquier dato que ha sido confiado a los profesionales durante la evaluación, diagnóstico y tratamiento. Protección similar se otorga a participantes de investigaciones y quienes responden encuestas respecto del acceso no autorizado a la información que revelan en confidencia" (23:104).

Finalmente, el cuarto concepto en juego en este análisis, corresponde al "anonimato", cuyo origen etimológico significa "sin nombre". Anguita y Sotomayor enfatizan que tanto confidencialidad como anonimato refieren a información "que se considera susceptible de protección, en razón de que su conocimiento por terceros puede dañar al titular de la misma"(16:200).

Será, no obstante, en el contexto de la investigación científica que el concepto adquiere su mayor relevancia; conlleva un tratamiento de los datos obtenidos en el curso de esta, generalmente concebido como "dato estadístico", aquel que "en su origen, o como consecuencia de su tratamiento, no puede ser asociado a un titular identificado o identificable" (Ley 19.628, Sobre la protección de la vida privada).

El cuidado del anonimato ha sido abordado frecuentemente en relación con muestras biológicas en la investigación biomédica, de las cuales se han eliminado los identificadores directos o indirectos, de tal manera que sea imposible establecer un vínculo entre los registros o muestras y la identidad de la persona de quien procede el registro o la muestra(20).

Se aprecia entonces la relevancia de tener claridad respecto de los derechos de las personas, del control que tienen sobre su información personal, así como de las obligaciones de profesionales y científicos en cuanto a proteger la información a la que tiene acceso en el ejercicio de sus funciones, sean estas para la construcción de conocimiento - como en la tarea científica- o para el otorgamiento de servicios especializados — como en la atención profesional-.

\section{Confidencialidad en la práctica profesional en salud mental}

El tópico de la confidencialidad ha permeado el ejercicio profesional en salud desde sus inicios; presente en el juramento hipocrático, se encuentra vigente en las diversas dimensiones en que se abor- 
da salud y la enfermedad, a lo largo de la historia y en distintas culturas.

Se trata, además, de un compromiso afectado en forma importante por los avances tecnológicos y científicos, así como por el desarrollo de las comunicaciones y la intromisión de las formas digitales de comunicación en la vida cotidiana del siglo XXI. Con Lustgarden(24) coincidimos en que el crecimiento tecnológico aumenta la vulnerabilidad de la privacidad de las personas, por lo que el tema de la protección de datos está cambiando y requiere precauciones y consideraciones éticas especiales.

En distintas profesiones, en función de los objetivos y características de su práctica, la cuestión de la confidencialidad adquiere dimensiones y formas propias, dependientes también del contexto en que se ejerce.

Un caso clásico y paradigmático en el campo de la salud mental —el caso Tarasoff, ocurrido en la década de los '70 en California- es referencia obligada en este análisis. Prosenjit Poddar, estudiante indio de la Universidad de Berkeley y perteneciente a una casta intocable, se involucró afectivamente con Tatiana Tarasoff, incumpliendo las normas que le imponía su pertenencia a la casta. Cuando Tatiana terminó la relación, que tuvo para ella un carácter de menor compromiso que para él, Poddar le comentó a su terapeuta que la mataría. Terminada la sesión, el terapeuta informó a la policía del campus de las intenciones de su paciente; la policía lo interrogó y Poddar negó que fuese a agredir a Tatiana, quedando en libertad. Posteriormente, fue a casa de Tatiana y la mató. Después de un largo juicio, en que los padres demandaron al terapeuta, a la policía y a la universidad, la Corte Suprema estableció que el psicoterapeuta tiene el deber de proteger a las potenciales víctimas de sus pacientes, si esa conducta presenta un riesgo serio de violencia, y que la protección consiste en prevenir a la víctima potencial; es decir, en este caso, no fue suficiente con informar a la policía universitaria, sino que debería haber informado a Tatiana Tarasoff del riesgo que corría(25).

A partir de dicho fallo ha surgido un largo debate, aún no resuelto, respecto del dilema que impone a los psicoterapeutas la norma Tarasoff. La postura más radical es de Bersoff, al plantear que la ley Tarasoff es "mala ley, mala ciencia y mala política social..." (26:461), ya que pone en riesgo la esencia misma de la psicoterapia, es decir la confianza que los pacientes deben tener en sus terapeutas, justamente para confidenciar sus más íntimos pensamientos, temores, deseos. Así, si una ley obliga a traicionar esa confianza, la posibilidad misma de la confianza es amenazada; y con ello las posibilidades de efectividad del trabajo terapéutico. A nivel legislativo el tema no está resuelto; aunque, por ejemplo, en Estados Unidos de Norteamérica se ha legislado estableciendo algunos privilegios entre psicoterapeuta y paciente, como se establece en la siguiente afirmación:

"Psicoanálisis y psicoterapia dependen de la revelación más completa de los detalles más íntimos y vergonzosos de la vida del paciente (...) aunque la garantía del privilegio puede operar en casos particulares para retener información relevante, el interés de la sociedad estará mejor resguardado si los psiquiatras son capaces de asegurar a sus pacientes que sus confidencias serán protegidas" (27:1032).

Desde Tarasoff la ley estadounidense es explícita respecto de las situaciones en que los profesionales están, no solo dispensados de resguardar la confidencialidad, sino obligados a informar. En nuestro país la situación es mucho menos clara, ya que no existe legislación específica que regule el ejercicio profesional de la psicología, por lo que cada profesional deberá recurrir a normas generales o referidas a otras profesiones para decidir cursos de acción en casos específicos.

En el caso del ejercicio de la medicina en Chile, el nuevo Código de Ética (2013) establece en su articulo 29: "El secreto profesional es un deber inherente al ejercicio de la profesión médica y se funda en el respeto a la intimidad del paciente, quien devela información personal, en la medida que ésta es útil para el tratamiento de su enfermedad". Y el artículo 31 especifica: "El médico debe guardar confidencialidad de toda información relativa a su paciente, ya sea que la obtenga de un relato verbal de aquél, o en virtud de los exámenes o intervenciones quirúrgicas que le practique. El secreto profesional incluye, además, el nombre del paciente y constituye para el médico una obligación que 
debe respetar, incluso, después de concluidos sus servicios profesionales, o una vez fallecido el paciente"(28).

No obstante, es posible detectar inconsistencias normativas, ya que la ley 20.584, que regula los derechos y deberes de las personas en relación con acciones vinculadas a su atención en salud (2012), establece que la ficha clínica contiene datos sensibles (artículo 12), que se encuentran protegidos; no obstante, en el artículo 13, la misma ley establece que “... copia de la ficha (...) será entregada (...) a) al titular de la ficha, a su representante legal o, en caso de fallecimiento del titular, a sus herederos". Este carácter de "herencia" que adquiere la ficha clínica no respeta la voluntad del paciente y su derecho al secreto, y al mismo tiempo releva nuevos problemas, muy específicamente en el caso de la ficha psicológica: se libera el secreto profesional, sin precauciones especiales respecto de los eventuales efectos de tal revelación en la propia familia.

Ahora bien, nuevos desarrollos tecnológicos en el campo de la atención en salud, como el aumento creciente del uso de tecnologías comunicacionales en la atención clínica, médica o psicológica $(29,30)$, han incrementado el riesgo de comprometer la confidencialidad del paciente(31) y obligan a reevaluar las normas éticas(24) en aspectos de contrato con clientes, competencia, confidencialidad y control de la práctica profesional(32).

En otros países se ha suscitado una larga controversia acerca de los beneficios del registro digital de los datos de salud versus los riesgos, referidos inicialmente a la pérdida de la confidencialidad y el riesgo de estigmatización, vergüenza u otros. Respecto de la formación profesional, en Estados Unidos de Norteamérica se ha documentado ventajas y riesgos que enfrentan estudiantes para ejercer la psicología clínica en el intercambio de información digital con otros profesionales(33), destacando la difícil posición que implica estar, al mismo tiempo, aprendiendo a utilizar fichas digitales y simultáneamente formándose en los temas de confidencialidad.

En un análisis de mayor alcance, a partir de las normas y estándares australianos se analiza los aspectos éticos involucrados en el uso de servicios psicológicos con tecnologías comunicacionales: contrato con clientes, competencia, confidencialidad y control de la práctica psicológica, concluyendo que se requiere una mirada interdisciplinaria de los riesgos éticos asociados a los desarrollos tecnológicos, junto con una evaluación crítica del contexto(34).

También han sido abordados el uso de registros digitales en hospitales infantiles, en psicología pediátrica(30), razones o justificaciones para quebrantar la confidencialidad, cuándo existe la obligación de prevenir e informar(27), la aplicabilidad de la terapia cognitiva en forma electrónica, en cuanto a costo, manejo de la confidencialidad, resguardo de registros, evaluación de riesgos y rol del terapeu$\operatorname{ta}(32)$, entre otros.

En una investigación(29) en la que los autores encuestaron a 141 psicólogos y estudiantes de psicología, a través de la web, en España, acerca del uso de la tecnología en comunicaciones, así como acerca de posibles brechas en la seguridad, los resultados mostraron que entre un tercio y la mitad de los clínicos reportaron comunicarse electrónicamente con pacientes intercambiando emails y mensajes de texto. Dependiendo del método, desde un quinto hasta la mitad informaron usar métodos inseguros, que pueden comprometer la confidencialidad-anonimato del paciente.

Los antecedentes presentados sustentan la necesidad de enriquecer la discusión acerca de ventajas y desventajas del uso de sistemas de registro y comunicaciones electrónicas, con investigación que aborde, en particular, los efectos en el trabajo clínico y el quiebre de la confidencialidad en los/as propios pacientes.

\section{Confidencialidad en la investigación científica: complejidades y desafíos actuales}

A diferencia de lo que ocurre en la práctica profesional, en que la información sensible o confidencial tiene por finalidad cardinal el tratamiento y bienestar del paciente, en el caso de la investigación científica la información que se obtiene tiene como objetivo final ser difundida y publicada. Ello, por supuesto, conlleva complejidades adicionales y exigencias específicas para el resguardo de dicha información, constituyendo confidenciali- 
dad y anonimato dos deberes de máximo valor.

En este contexto, importa que el avance de la ciencia dependa de participantes que, voluntariamente, acepten entregar información acerca de sus creencias y acciones en una gran cantidad de temas; considerando que un participante estará más dispuesto a entregar información en forma honesta si su identidad no está expuesta.

Actualmente, la principal controversia gira en torno al deber de contar con el consentimiento informado de los pacientes para el uso de sus datos de salud-enfermedad con fines de investigación científica. Como se ha señalado, el consentimiento informado adquiere un valor central en la práctica profesional actual y, a diferencia de lo que ocurría hace algunas décadas, hoy existen varias restricciones explícitas para el uso de dicha información.

Una perspectiva se centra en los riesgos que conlleva la protección estricta de la confidencialidad, en tanto puede obstruir el logro de la investigación médica y el uso de los registros médicos electrónicos(31). Por ejemplo, Melton(35) informa que el uso de cinco millones de registros médicos produjo cientos de investigaciones y miles de publicaciones respecto del curso natural de ciertas enfermedades, y que solo fue posible porque una ley en Minnesota permite la liberación de registros médicos para investigación científica sin el consentimiento expreso del paciente. Este constituye un caso que previene de los riesgos que conlleva exigir consentimiento del titular de los datos para toda investigación.

Este vigoroso debate entre científicos acerca de la posible pérdida de resultados e información relevante ante la exigencia del consentimiento informado, implica la pregunta respecto de qué tipos de derechos deben prevalecer: fortalecer el derecho al consentimiento informado enfatiza los derechos individuales; preferir el derecho al conocimiento fortalece los derechos sociales o de la comunidad.

\section{Conclusiones}

Revisitar el tema de la confidencialidad en el contexto actual evidencia un conjunto de desafíos y nuevos retos que profesionales y científicos deben enfrentar. En particular, los avances en los medios de comunicación, la ficha clínica electrónica, las posibilidades de comunicación digital entre profesional y paciente y entre participante e investigador configuran un contexto profesional y científico nunca antes conocido, que obliga a la revisión de prácticas, normas y estándares que regulen tales relaciones.

Las violaciones a la confidencialidad constituyen un atentado a la dignidad humana; la invasión a la privacidad ofende el respeto y sensación de control de la persona(20). Las posibilidades de daño varían desde sentirse avergonzado hasta la estigmatización; incluso puede haber daño al estatus social y económico, como la pérdida de la fuente laboral o del seguro de salud.

Así, la privacidad, esto es, el interés o el derecho de las personas o de los grupos pequeños, y la confidencialidad, que constituye una obligación de los profesionales, se encuentra en riesgo en la investigación en salud. Cuando la privacidad y la confidencialidad pueden estar totalmente protegidas sin comprometer el valor científico de la investigación, es indudable que los científicos tienen la obligación de protegerlas, superando cualquier obstáculo.

Situaciones de riesgo específicas emergen tanto del propio sistema de registro de información en salud, como de algunas características y condiciones de pacientes en atención y participantes en investigación científica. Por ejemplo, en el sistema de salud público chileno, el acceso en todo el país a fichas clínicas que tiene el personal en salud. Si bien la ley exige confidencialidad de la ficha, ello no protege del riesgo inherente a la circulación de información. Otro tanto puede señalarse para menores de edad y personas interdictas respecto de quienes ejercen como tutores, y el equilibrio que es necesario mantener entre su intimidad y el conocimiento de información crítica para asegurar su protección por parte de estos últimos.

Se hace evidente, entonces, la necesidad imperiosa de transformar el resguardo de la intimidad y la promesa de confidencialidad en un tema de estudio y debate en Chile. Al mismo tiempo, se requiere actualizar los planes de formación de profesionales y científicos, particularmente en salud mental, incorporando reflexiones ético-legales que 
emergen en los nuevos contextos de investigación y desempeño profesional. Transversal a ambos campos - práctica profesional e investigaciónes la relevancia de complejizar el proceso de consentimiento informado, en sus dimensiones de derecho y deber.

Considerando la discusión en torno al tema de la confidencialidad, desde las limitaciones que impuso el caso Tarasoff en Estados Unidos hasta los riesgos que conlleva el uso de fichas electrónicas, nos sumamos a los planteamientos de Mulligan y Braunack-Mayer(31:48): "la confidencialidad deber seguir siendo protegida para todos los pacientes (...) porque la provisión de servicios de salud confidenciales protege a los pacientes de daño, apoya el acceso al cuidado en salud y produce mejores resultados en salud".

\section{Agradecimientos}

Este artículo es producto del proyecto SOC 1110, modalidad Anillos de Investigación en Ciencias Sociales, PIA-Conicyt. Los autores agradecen el apoyo recibido a Conicyt, así como a la Universidad de Santiago de Chile, a través de la Vicerrectoría de Investigación, Desarrollo e Innovación, Vridei.

\section{Referencias}

1. Bauman Z. Tiempos Líquidos. Barcelona: Tusquets Editores; 2007.

2. Beck U. La Sociedad del Riesgo: hacia una Nueva Modernidad. Barcelona: Paidós; 1998.

3. Rovaletti ML. Derechos Humanos, Sociedad de la Información y Sociedad de Riesgo. Acta Bioethica 2010; 16(2): 174-179.

4. Cano F. El consentimiento bajo información ¿un documento o un proceso? Bioética. México: UNAM; 2005: 23-33.

5. Kitcher Ph. Science, Truth and Democracy. Oxford: Oxford University Press; 2001.

6. Kitcher Ph. Science in a democratic Society. New York: Prometheus Books; 2011.

7. Álvarez F. Responsabilidad, confianza y modelos humanos. Isegoría 2003; 29: 51-68. DOI:10.3989/isegoria.2003. i29.488

8. Valcárcel M, Lucerna R. Responsabilidad social de la ciencia y la tecnología. Anales de Química 2012; 108(2), 156-159.

9. Valero J. Responsabilidad social de la actividad científica Revista Internacional de Sociología 2006; LXIV(43): 219-242.

10. Kant I. Fundamentación de la metafísica de las costumbres. Barcelona: Ariel; 2000.

11. Aguiar F. Confianza y racionalidad. Educação e Filosofia 1994; 8(16): 239-245.

12. Nietzsche F. Genealogía de la moral. Madrid: Alianza; 1995.

13. Hume D. Investigación sobre los principios de la moral. Madrid: Alianza; 2006.

14. Canto-Sperber M. Diccionario de Ética y Filosofía moral, Tomo I. Madrid: Fondo de Cultura Económica; 2001.

15. Luhmann N. Confianza. Madrid: Anthropos; 2005.

16. Anguita MV, Sotomayor MA. ¿Confidencialidad, anonimato?: las otras promesas de la investigación. Acta Bioethica 2011; 17(2): 199-204. DOI: http://dx.doi.or/10.4067/S1726-569X2011000200006.

17. García Sanz J. El Secreto Profesional en el Ámbito Sanitario. En Benítez IF, Morillas L, Peris JM. (coords.) Estudios Jurídico-penales sobre Genética y Biomedicina: Libro Homenaje al Prof. Dr. D. Ferrando Mantovani. Madrid: Dykinson; 2005: 459-481.

18. Bajo Fernández M. El Secreto Profesional en el Proyecto de Código Penal. Anuario de Derecho Penal y Ciencias Penales 1980; 595-610.

19. López Díaz E. El derecho al honor y el derecho a la intimidad. Jurisprudencia y doctrina. Madrid: Dykinson; 1996.

20. Cash R, Wikler D, Saxena A, Capron A. Estudios de Caso sobre Ética de la Investigación Internacional en Salud. Washington, DC: OPS/OMS; 2014.

21. Sánchez Carazo C. La Intimidad y el Secreto Médico. Madrid: Díaz de Santos; 2000.

22. Sánchez Caro J. El Médico y la Intimidad. Madrid: Díaz de Santos; 2001: 74.

23. American Psychological Association. Ethical Principles of Psychologists and Code of Conduct; 2010. Recuperado de: http: www.apa.org/ethics/code. 
24. Lustgarden S. Emerging Ethical Threats to Client Privacy in Cloud Communication and Data Storage. Professional Psychology: Research and Practice 2015; 46(3): 154-160.

25. Tarasoff v. Regents of the University of California (1976). Public Health and Ethics: A Reader 2002: 1-30.

26. Bersoff D. Protecting Victims of Violent Patients While Protecting Confidentiality. American Psychologist 2014; 69(5): 461-467 DOI: http://dxdoi.org/10.1037/a0037198.

27. Fleming J, Maximov B. The Patient or His Victim: The Therapist's Dilemma. 62. California Law Review 1974; 62(3): 1025-1068. DOI: http://dx.doi.org/doi:10.15779/Z38X17M.

28. Colegio Médico de Chile (A.G.). Código de Ética. Disponible en: http://www.colegiomedico.cl/wp-content/ uploads/2015/06/colmed_codigo_etica_2013.pdf.

29. Elhai J, Hall B. How secure is mental health providers' electronic patient communication? An empirical investigation. Professional Psychology: Research and Practice 2015; 46(6): 444-450. DOI: http://dx.doi.org/10.1037/pro000054.

30. Nielsen B. Confidentiality and electronic health records: Keeping up with advances in technology and expectation for access. Clinical Practice in Pediatric Psychology 2015; 3(2): 175-178. DOI: http://dx.doi.org/10.1037/cpp0000096.

31. Mulligan E, Braunack-Mayer A. Why protect confidentiality in health records? A review of research evidence. Australian Health Review 2004; 28(1): 48-55.

32. Lawlor-Savage L. Prentice L. Digital Cognitive Behavior Therapy (CBT) in Canada: Ethical considerations. Canadian Psychology 2014; 55(4): 231-239. DOI: http://dx.doi.org/10.1037/a0037861.

33. Wallace C. Electronic Health Records: A graduate student's experience. Clinical Practice in Pediatric Psychology 2015; 3(2): 179-181. DOI: http://dx.doi.org/10.1037/cpp000098.

34. Davis A. Ethical issues for psychologists using communication technology: An Australian perspective on service provision flexibility. Professional Psychology: Research and Practice 2014; 45(5): 303-308. DOI: http://dx.doi.org/10.1037/ a0037081.

35. Melton J. The threat to medical-records research. New England Journal of Medicine 1997; 337: 1466-1470.

Recibido: 11 de julio de 2016

Aceptado: 24 de agosto de 2016 